\title{
Phrenic nerve palsy during ablation of atrial fibrillation using a 28-mm cryoballoon catheter: predictors and prevention
}

\author{
Michael Kühne • Sven Knecht • David Altmann • \\ Nadine Kawel • Peter Ammann • Beat Schaer • \\ Stefan Osswald $\cdot$ Christian Sticherling
}

Received: 1 June 2012 / Accepted: 14 September 2012 /Published online: 23 October 2012

(C) Springer Science+Business Media New York 2012

\begin{abstract}
Purpose The purposes of this study were to determine whether predictors of phrenic nerve palsy (PNP) exist and to test whether a standardized ablation protocol may prevent PNP during cryoballoon (CB) ablation using the $28 \mathrm{~mm} \mathrm{CB}$.

Methods Three-dimensional (3D) geometry of the pulmonary veins (PV) and their relationship to the superior vena cava (SVC) was analyzed. Phrenic nerve (PN) stimulation was performed during ablation of the right-sided PVs with a 28-mm CB. The freezing cycle was immediately terminated in case of loss of PN capture.

Results Sixty-five patients (age, $58 \pm 11$ years; ejection fraction, $0.59 \pm 0.06$; left atrial size, $40 \pm 5 \mathrm{~mm}$ ) with paroxysmal atrial fibrillation were included. No persistent PNP was observed. Transient PNP occurred in 4 of 65 patients (6 \%). PN function normalized within $24 \mathrm{~h}$ in all four patients. A short distance between the right superior PV and the SVC was significantly associated with PNP, but left atrial and 3D PV anatomy were not. Low temperature early during the freezing cycle $\left(<-41{ }^{\circ} \mathrm{C}\right.$ at $\left.30 \mathrm{~s}\right)$ predicted PNP with a sensitivity and a specificity of 100 and $98 \%$, respectively.
\end{abstract}

M. Kühne and S. Knecht contributed equally to this manuscript

M. Kühne $(\bowtie) \cdot$ S. Knecht $\cdot$ D. Altmann · B. Schaer •

S. Osswald $\cdot$ C. Sticherling

Division of Cardiology, University of Basel Hospital,

Petersgraben 4,

4031, Basel, Switzerland

e-mail:kuehnem@uhbs.ch

N. Kawel

Department of Radiology, University of Basel Hospital,

Basel, Switzerland

P. Ammann

Division of Cardiology, Kantonsspital St. Gallen,

St. Gallen, Switzerland
Conclusion The anatomical relationship between the right superior PV and the SVC is a preprocedural predictor for the development of transient PNP, and low temperature early during ablation at the right superior $\mathrm{PV}$ is a sensitive warning sign of impending PNP. Despite the use of the $28 \mathrm{~mm} \mathrm{CB}$, transient PNP occurred in $6 \%$ of patients undergoing $\mathrm{CB}$ ablation.

Keywords Phrenic nerve palsy · Cryoballoon ablation . Atrial fibrillation $\cdot$ Pulmonary vein isolation

\section{Introduction}

Pulmonary vein (PV) isolation (PVI) has evolved tremendously over the last decade and is regarded as the cornerstone of interventional treatment of atrial fibrillation (AF) using catheter ablation [1-3]. Cryoballoon (CB) ablation is a balloon-based treatment modality for patients with $\mathrm{AF}$ allowing the creation of potentially continuous circumferential lesion sets around the PVs [4-7]. Due to the close proximity of the right-sided $\mathrm{PVs}$ to the right phrenic nerve (PN), phrenic nerve palsy (PNP) has been reported in 5$10 \%$ of patients, and the use of the $23 \mathrm{~mm}$ balloon catheter seems to be associated with a higher incidence of PNP [5, 8, 9]. Important factors for the occurrence of PNP when performing $\mathrm{CB}$ ablation may be the anatomy of the PVs and the PN, the diameter of the balloon used, and temperatures reached during ablation, but this has not been studied systematically. The course of the right PN is generally anterior to the right superior PV (RSPV) at the lateral aspect of the superior vena cava (SVC), and the nerve may be stimulated cranial to the ablation site from a catheter positioned in the SVC [10]. The purposes of this study were to determine whether anatomical or procedural predictors of PNP during CB ablation of the right-sided PVs exist and to 
test whether a standardized protocol with the systematic use of high-output right PN stimulation in conjunction with a single 28-mm CB catheter could prevent PNP.

\section{Methods}

\subsection{Study population}

The subjects of this study were 65 patients with symptomatic drug-refractory paroxysmal AF. The presence of intracardiac thrombus was ruled out by transesophageal echocardiography before the procedure. All patients underwent cardiac magnetic resonance or computed tomography to assess left atrial and PV anatomy with three-dimensional reconstruction.

\subsection{Image acquisition}

Magnetic resonance imaging was performed on a 1.5-T scanner (MagnetomAvanto/ Espree, Siemens, Germany) equipped with phased array body coils. Routine multiplanar scout images were acquired. A respiratory- and electrocardiography (ECG)-gated three-dimensional balanced steadystate free precession sequence was acquired in axial orientation covering the left atrium. A navigator was placed perpendicular to the liver/lung interface enabling breathgating during free breathing.

Computed tomography studies were performed on a 64detector row CT scanner (Sensation 64, Siemens, Germany). Eighty milliliters i.v. contrast agent was administered with a flow rate of $2.5 \mathrm{~mL} / \mathrm{s}$ followed by a saline chaser. Image acquisition was started after a delay of $28 \mathrm{~s}$. Prospective ECG gating was used acquiring images at mid-diastole.

\subsection{Image analysis}

Geometry of the left atrium and the SVC was segmented using the CartoMerge software (Biosense Webster, Diamond Bar, USA) and analyzed in three dimensions (3D). The long and short axes of the PV at the ostium and its perimeter were measured using an electronic caliper provided by the software. The ovality index of the PV ostia was calculated for all veins by dividing the short axis by the long axis (Fig. 1). Furthermore, the shortest distance between the ostium of the right superior PV and the lateral wall of the SVC (RSPV-SVC distance) as a surrogate for the distance between the ablation site and the right PN was measured (Fig. 2) [10].

\subsection{Cryoballoon ablation}

Informed consent was obtained from all patients. All procedures were performed under conscious sedation. Venous

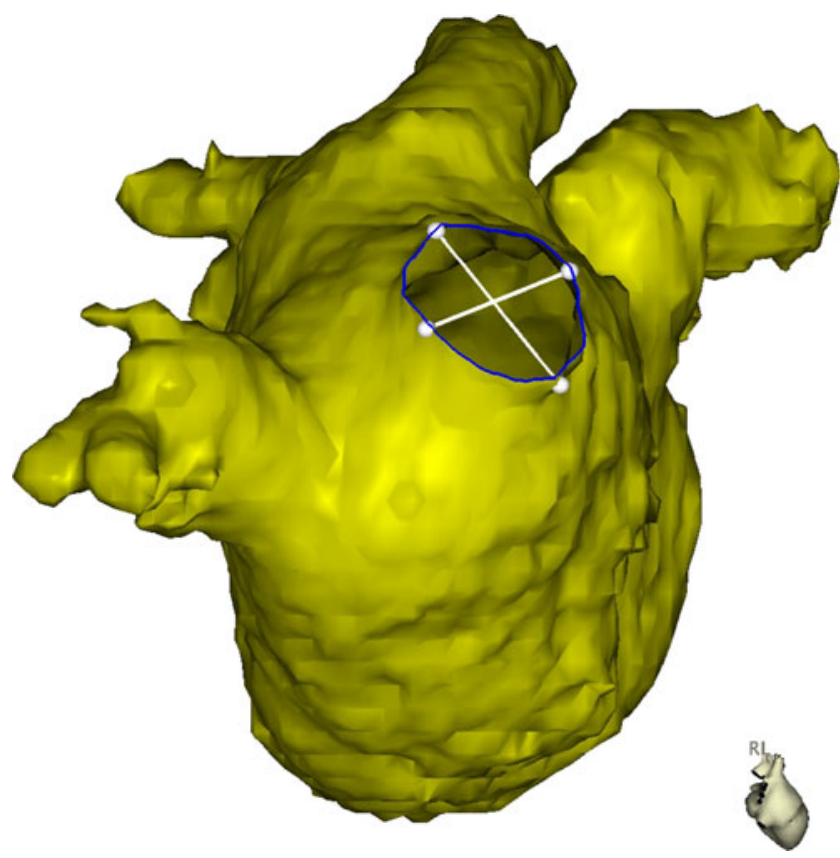

Fig. 1 MRI reconstruction of the left atrium in a right lateral view. Measurements for diameter in the long and short axis and the perimeter of the right pulmonary veins were performed in $3 \mathrm{D}$

access was obtained via the right femoral vein. One catheter was positioned in the coronary sinus as a reference and for pacing. Double transseptal puncture was performed under fluoroscopic guidance. Intravenous heparin was used to achieve an activated clotting time of 350 s. A 20-pole circumferential mapping catheter (Lasso, Biosense Webster, Diamond Bar, USA) was advanced into the left atrium. The intracardiac and surface electrograms were displayed on an oscilloscope and recorded at a speed of $100 \mathrm{~mm} / \mathrm{s}$.

A steerable sheath (FlexCath, Medtronic, USA) was advanced through the second transseptal puncture through which the 28-mm cryoballoon (Arctic Front, Medtronic, USA) was advanced into the left atrium. A freezing cycle with a standard duration of $300 \mathrm{~s}$ was started after PV occlusion was demonstrated by contrast injection. Occlusions were assessed using a semiquantitative grading system (grades 1-4) [5]. Continuous temperature measurements (sampling rate, 1/s) were obtained from the sensor located at the proximal end of the balloon. The circumferential mapping catheter was inserted into the PV to assess for isolation after two freezing cycles and then after each freezing cycle if additional applications were necessary to achieve PVI. The endpoint of the ablation was the elimination of all PV potentials on the circumferential mapping catheter. If ablation using the $\mathrm{CB}$ was not effective in isolating the PV after up to a maximum of six applications per vein, PVI was completed using an irrigated-tip radiofrequency ablation catheter [7]. 


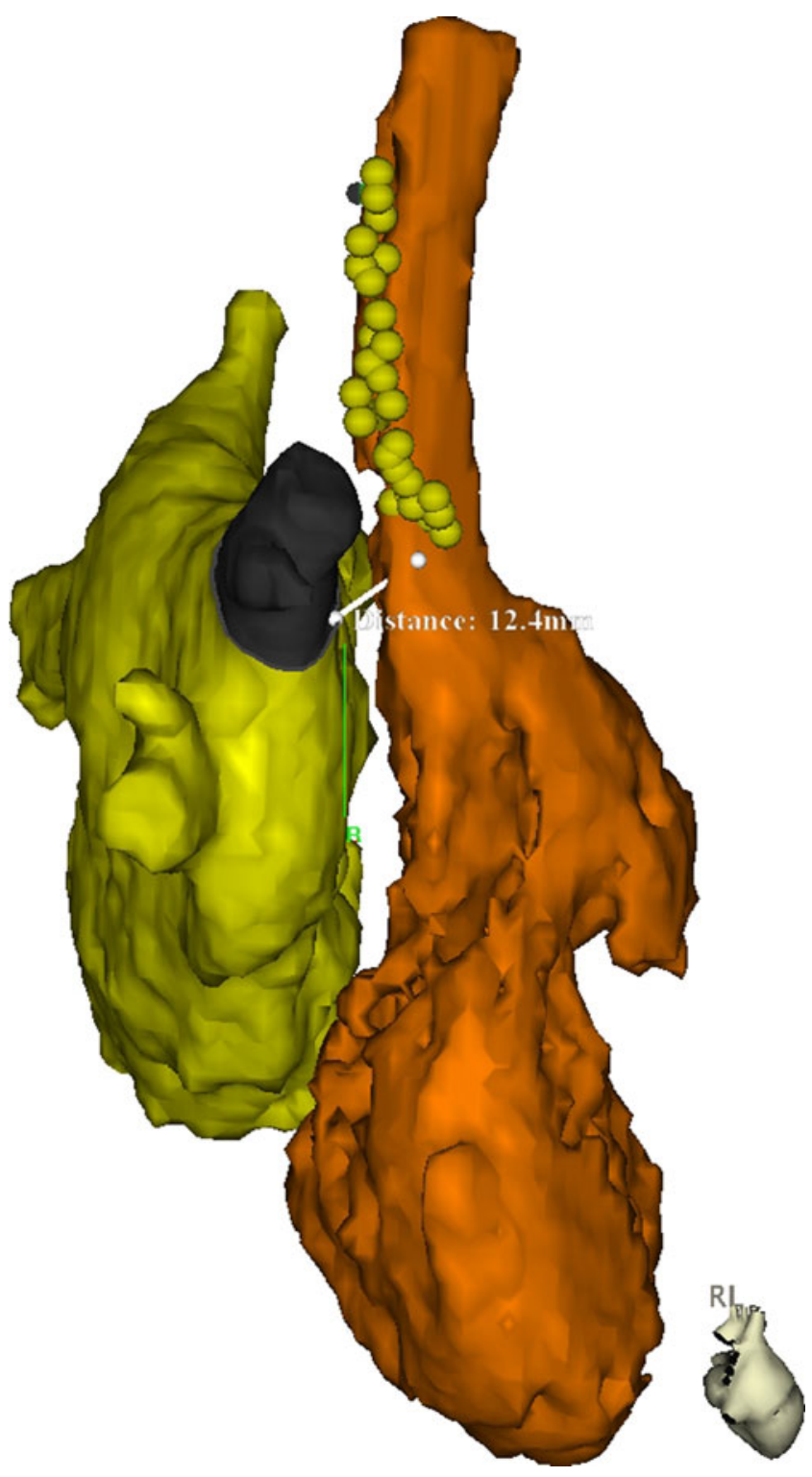

Fig. 2 MRI reconstruction of the left (yellow) and right (orange) atrium in a right lateral view showing the course of the right phrenic nerve in a random patient undergoing ablation of atrial fibrillation. The yellow tags denote sites with phrenic nerve capture (as a marker of the course of the phrenic nerve) using a catheter located in the superior vena cava. The indicated measurement shows the distance between the ostium of the right superior PV and the lateral wall of the SVC (RSPV-SVC distance)

\subsection{Phrenic nerve stimulation}

Because of reports of PNP after ablation at the right-sided PVs especially with the use of the $23 \mathrm{~mm}$ balloon, only the 28-mm diameter CB was used [5,9]. A catheter was positioned in the SVC for PN stimulation. Right PN capture was confirmed by fluoroscopy and manual confirmation of diaphragmatic stimulation before balloon positioning at the right-sided PVs. After balloon inflation and confirmation of PV occlusion by contrast injection, the freezing cycle was started. PN stimulation was initiated $20 \mathrm{~s}$ after the freezing cycle was started. This approach was used because movements of the right diaphragm during PN stimulation may result in an unstable CB catheter position. During ablation at both the right superior and the right inferior PV, the right PN was stimulated with an output of $12 \mathrm{~V} / 2.9 \mathrm{~ms}$ at a cycle length of $1,000 \mathrm{~ms}$, and continuous capture was confirmed manually during the freezing cycle. In case of loss of PN capture or decrease in diaphragmatic contraction, the freezing cycle was immediately terminated. Figure 2 illustrates the course of the right $\mathrm{PN}$ in a random patient undergoing $\mathrm{AF}$ ablation.

\subsection{Postablation management and follow-up}

Oral anticoagulation was continued for at least 3 months. All antiarrhythmic drugs were stopped after the procedure. Follow-up consisted of outpatient clinic visits at 3, 6, and 12 months and included a detailed history, physical examination, 12-lead ECG, 24-h Holter monitoring, and a 7-day monitor at 12 months. Episodes of AF (>30 s) were counted as recurrences. Recurrence rates were analyzed with a postprocedural blanking period of 3 months [3].

\subsection{Statistical analysis}

Continuous variables are presented as mean \pm one standard deviation or as median and interquartile range (IQR) in case of skewed distribution. For continuous variables, comparisons were made using Student's $t$ test, or Mann-Whitney $U$ test, as appropriate. Discrete variables were compared using Fisher's exact test. A $p<0.05$ was considered to indicate statistical significance. Calculations were made using GraphPad Prism (5.0 a).

\section{Results}

\subsection{Clinical characteristics of the patients}

The study population consisted of 65 patients referred for ablation of paroxysmal AF ( $74 \%$ male, age $58 \pm 11$ years). The mean left ventricular ejection fraction was $0.59 \pm 0.06$; left atrial size was $40 \pm 5 \mathrm{~mm}$ (parasternal long-axis). Fortyseven of 65 patients $(72 \%)$ had no structural heart disease. Baseline characteristics are shown in Table 1.

\subsection{Anatomy of the right-sided pulmonary veins}

The mean diameters in the long and short axes of the right superior PV were $21 \pm 3 \mathrm{~mm}$ and $16 \pm 3 \mathrm{~mm}$, respectively. The ovality index was $0.79 \pm 0.11$, and the perimeter was $58 \pm$ 
Table 1 Patient characteristics

\begin{tabular}{ll}
\hline Number of patients & 65 \\
Age (years)—mean \pm SD & $58 \pm 11$ \\
Men— $n(\%)$ & $48(74)$ \\
Duration of AF (months)—median (IQR) & $54(22-87)$ \\
Episodes of AF & \\
Daily-n(\%) & $34(52)$ \\
$>1$ episode/week-n(\%) & $23(35)$ \\
$<1$ episode/week-n(\%) & $8(12)$ \\
Left atrial size (mm)—mean \pm SD & $40 \pm 5$ \\
Left ventricular ejection fraction $(\%)$-mean \pm SD & $59 \pm 6$ \\
No structural heart disease— $n(\%)$ & $47(72)$ \\
HCVD— $n(\%)$ & $13(20)$ \\
CAD— $n(\%)$ & $5(8)$ \\
\hline
\end{tabular}

$I Q R$ interquartile range, $H C V D$ hypertensive cardiovascular disease, $C A D$ coronary artery disease

$10 \mathrm{~mm}$. For the right inferior PV, the long and short axes were $20 \pm 3 \mathrm{~mm}$ and $17 \pm 3 \mathrm{~mm}$, the ovality index and the perimeter were $0.88 \pm 0.08$ and $58 \pm 9 \mathrm{~mm}$, respectively. The ovality index of the right superior PV was significantly lower (more oval) compared to the right inferior PV $(p<0.0001)$.

For the assessment of intraobserver variability, the technical error of measurements was $1.08 \mathrm{~mm}, 0.80 \mathrm{~mm}, 0.04$, and $3.54 \mathrm{~mm}$ for the right superior PV long axis, short axis, ovality index, and perimeter, respectively. For the respective measurements, technical error of measurements was $1.25 \mathrm{~mm}$, $1.18 \mathrm{~mm}, 0.05$ and $2.86 \mathrm{~mm}$ for the right inferior PV, respectively.

\subsection{Procedural data}

The mean procedure, fluoroscopy, and ablation times are given in Table 2. The procedural endpoint of PVI was reached in all 65 patients. Of the targeted PVs, 143 were right sided (including 13 right middle PVs). Of these, 126 PVs (88\%) could be isolated using the CB alone, the remaining $17 \mathrm{PVs}(12 \%)$ were isolated using the $3.5-\mathrm{mm}$ irrigated-tip ablation catheter. Occlusion grades of the right superior and inferior PV were $3.8 \pm 0.4$ and $3.3 \pm 0.8$, respectively $(p<0.0001)$. The median ablation time for the right superior, middle, and inferior PVs was 10 (IQR, 10-10), 11.5 (IQR, 10-13), and 10 (IQR, 10-15) min, respectively.

Table 2 Procedural data

\begin{tabular}{lr}
\hline Procedure time (minutes) — mean \pm SD & $165 \pm 35$ \\
Fluoroscopy time (minutes)—-mean \pm SD & $45 \pm 21$ \\
Ablation time (minutes)—-mean \pm SD & $46 \pm 10$ \\
\hline
\end{tabular}

\subsection{Phrenic nerve palsy}

PNP during ablation at the right superior PV occurred in 4 of 65 patients $(6 \%)$ and resulted in immediate termination of the freezing cycle (within $2 \mathrm{~s}$ after detection). In three cases of PNP, resumption of PN function was immediate (within $30 \mathrm{~s}$ ). In the fourth case, partial resumption of PN function could be seen after $11 \mathrm{~min}$, and normal PN function was documented by fluoroscopy $24 \mathrm{~h}$ after the procedure. No cases of PNP occurred during ablation at the right middle or inferior PV. In the first case, PNP occurred after $67 \mathrm{~s}$; in the second case, after $178 \mathrm{~s}$; in the third case, after $108 \mathrm{~s}$; and in the fourth case, after $49 \mathrm{~s}$ (Fig. 3). In the first case, the PV was not isolated after the first freezing cycle, and isolation was achieved with a second freezing cycle with the $\mathrm{CB}$ in a more antral position. In the other three cases of PNP, the right superior PV was isolated after the freezing cycle leading to PNP.

PV anatomy (diameter, perimeter, and ovality index), grade of PV occlusion, and baseline characteristics (e.g., left atrial size, left ventricular ejection fraction) did not differ between patients with and without the occurrence of PNP. However, the distance between the ostium of the right superior $\mathrm{PV}$ and the lateral wall of the SVC (RSPV-SVC distance) was significantly shorter in patients with PNP (PNP group). Table 3 summarizes these findings. Furthermore, low temperatures were significantly associated with PNP. However, the comparison of the lowest temperatures reached in the PNP group and the nonPNP group underestimates the true difference because the freezing cycles were terminated early in the PNP group because of the occurrence of PNP. Since PNP occurred $49 \mathrm{~s}$ after the beginning of the freezing cycle or later, temperatures were compared at different time points early during the freezing cycle $(20,30$, and $40 \mathrm{~s}$ after the start of the ablation). These early temperature measurements showed significantly lower values in the PNP group (Table 4).

\subsection{Clinical outcomes after AF ablation}

With a postprocedural blanking period of 3 months and a second procedure using radiofrequency energy in 23 patients $(35 \%), 56$ of the 65 patients $(86 \%)$ were in stable sinus rhythm without antiarrhythmic drugs after a follow-up of $14 \pm 5$ months.

\subsection{Complications}

One of the 65 patients $(1.5 \%)$ had a postprocedural complication with the development of a large groin hematoma prolonging the patient's duration of stay in the hospital, but did not require surgical intervention. 

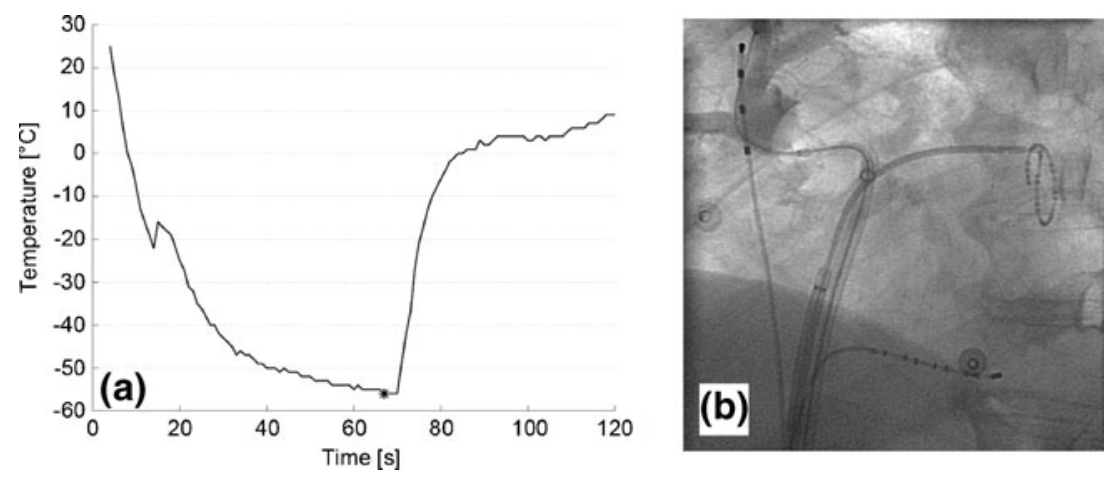

Fig. 3 (a) The course of the temperature during cryoballoon ablation at the right superior pulmonary vein is shown. Relatively low temperatures were reached early during the freezing cycle. Loss of phrenic nerve capture occurred after $67 \mathrm{~s}$ at a temperature of $-56^{\circ} \mathrm{C}$ (see asterisk). The freezing cycle was terminated immediately and resumption of phrenic nerve capture was immediate (within $10 \mathrm{~s}$ ). (b)

\section{Discussion}

\subsection{Main findings}

The main findings of this study are as follows: In a standard patient population with symptomatic paroxysmal AF undergoing ablation using the $\mathrm{CB}$ in conjunction with high-output $\mathrm{PN}$ stimulation at a rate of $60 \mathrm{bpm}$ and continuous manual confirmation of capture, transient PNP occurred in $6 \%$ of cases despite the use of the $28 \mathrm{~mm} \mathrm{CB}$. However, no PNP persisting beyond $24 \mathrm{~h}$ could be observed. A short RSPVSVC distance as a surrogate for the distance between the ablation site and the right PN was significantly associated with transient PNP, but PV anatomy was not. Furthermore, low temperatures during the freezing cycle were significantly associated with transient PNP.

Table 3 Pulmonary vein anatomy of the right-sided veins and left atrial size in patients with (PNP group) and without occurrence of phrenic nerve palsy (no PNP group)

\begin{tabular}{lccc}
\hline & $\begin{array}{l}\text { No PNP } \\
(n=61)\end{array}$ & $\begin{array}{l}\text { PNP } \\
(n=4)\end{array}$ & $P$ value \\
\hline Left atrial size (mm) & $39 \pm 5$ & $44 \pm 6$ & 0.13 \\
RSPV-SVC distance (mm) & $17.1 \pm 2.8$ & $14.0 \pm 2.3$ & 0.03 \\
RSPV diameter long (mm) & $20.7 \pm 3.5$ & $18.8 \pm 1.5$ & 0.30 \\
RSPV diameter short (mm) & $16.2 \pm 3.3$ & $16.0 \pm 2.5$ & 0.93 \\
RSPV perimeter (mm) & $57.9 \pm 10.0$ & $54.8 \pm 5.8$ & 0.54 \\
RSPV—ovality index & $0.78 \pm 0.11$ & $0.85 \pm 0.10$ & 0.25 \\
RIPV diameter long (mm) & $19.6 \pm 2.9$ & $18.5 \pm 1.6$ & 0.44 \\
RIPV diameter short (mm) & $17.3 \pm 3.2$ & $16.7 \pm 2.7$ & 0.73 \\
RIPV perimeter (mm) & $57.9 \pm 9.2$ & $55.2 \pm 6.3$ & 0.56 \\
RIPV—ovality index & $0.88 \pm 0.08$ & $0.90 \pm 0.10$ & 0.60 \\
\hline
\end{tabular}

$R S P V$ right superior pulmonary vein, $S V C$ superior vena cava, $R I P V$ right inferior pulmonary vein
Fluoroscopic image showing complete occlusion of the right superior PV (demonstrated by contrast injection) using a $28-\mathrm{mm}$ cryoballoon catheter. The guidewire is advanced into an upper branch of the right superior PV. The catheter in the superior vena cava for pacing, the circumferential mapping catheter, and the catheter in the coronary sinus can also be appreciated

\subsection{Impact of anatomy and temperatures reached during ablation}

There are limited data available with regards to where in the antrum ablation is actually performed when using the CB catheter. Van Belle et al. [11] performed electro-anatomical mapping in patients after undergoing 28-mm CB ablation and concluded that the majority of PVs undergo antral isolation. However, a minority of PVs with larger diameters was found to undergo ostial isolation. This is thought to be one aspect of the development of PNP because it may be that ablation is performed deeper within the vein resulting in lesions closer to the PN. Based on this hypothesis, anatomical variables such as the ones assessed in this study should be predictors of PNP. However, our data suggest that the preprocedurally assessed anatomical parameters such as left

Table 4 Pulmonary vein occlusions and temperatures reached during ablation at the right-sided pulmonary veins in patients with (PNP group) and without occurrence of phrenic nerve palsy (no PNP group)

\begin{tabular}{|c|c|c|c|}
\hline & $\begin{array}{l}\text { No PNP } \\
(n=61)\end{array}$ & $\begin{array}{l}\text { PNP } \\
(n=4)\end{array}$ & P-value \\
\hline RSPV temperature $\left({ }^{\circ} \mathrm{C}\right)$ & $-44 \pm 8$ & $-55 \pm 6$ & 0.008 \\
\hline RSPV occlusion — grades $1-4$ & $3.8 \pm 0.4$ & $4 \pm 0$ & $\mathrm{n} / \mathrm{a}$ \\
\hline $\begin{array}{l}\text { RSPV temperature } \\
20 \mathrm{~s} \text { after start }\left({ }^{\circ} \mathrm{C}\right)\end{array}$ & $-18 \pm 8$ & $-33 \pm 2$ & 0.0003 \\
\hline $\begin{array}{l}\text { RSPV temperature } \\
30 \mathrm{~s} \text { after start }\left({ }^{\circ} \mathrm{C}\right)\end{array}$ & $-28 \pm 8$ & $-44 \pm 2$ & 0.0003 \\
\hline $\begin{array}{l}\text { RSPV temperature } \\
40 \mathrm{~s} \text { after start }\left({ }^{\circ} \mathrm{C}\right)\end{array}$ & $-33 \pm 7$ & $-48 \pm 1$ & $<0.0001$ \\
\hline RIPV temperature $\left({ }^{\circ} \mathrm{C}\right)$ & $-38 \pm 9$ & $-43 \pm 5$ & 0.21 \\
\hline RIPV occlusion-grades $1-4$ & $3.2 \pm 0.8$ & $3.8 \pm 0.5$ & 0.20 \\
\hline
\end{tabular}

For the right superior pulmonary vein, temperatures reached early during the freezing cycle are given at different time points

$R S P V$ right superior pulmonary vein, $R I P V$ right inferior pulmonary vein, $n / a$ not applicable 
atrial size and PV anatomy do not suffice to predict the occurrence of PNP. The only anatomical parameter that was significantly associated with the occurrence of PNP was the relationship between the RSPV and SVC (RSPVSVC distance). This is a parameter that can be assessed during preprocedural imaging and could therefore have an impact on procedure planning because patients at higher risk for developing PNP could be excluded based on the MRI or CT. This also has financial implications because it may be that after a prematurely terminated freezing cycle because of PNP a switch to a radiofrequency ablation catheter may be necessary in order to complete PVI.

Based on the early reported experience of a higher rate of PNP with the $23 \mathrm{~mm}$ compared to the $28 \mathrm{~mm} \mathrm{CB}$, the exclusive use of the larger $28 \mathrm{~mm} \mathrm{CB}$ has been thought to minimize the incidence of PNP [12]. As in other studies, however, we could not eliminate the problem of PNP using solely the $28 \mathrm{~mm} \mathrm{CB}$ in our study, but PNP was only transient. The balloon-centric view on the anatomical relationship between both balloon sizes within a PV ostium confirms that the proper selection of the CB based on the mean PV diameter can reduce the risk of a more distal lesion also with the $23 \mathrm{~mm} \mathrm{CB}$ (Fig. 4).

Low temperatures were reached during the freezing cycle in all four patients in whom PNP occurred. Analysis of the temperature course within the first $40 \mathrm{~s}$ after the initiation of the ablation revealed that temperature dropped significantly more rapidly in patients with PNP. Therefore, assessment of the temperature early during the freezing cycle may be a

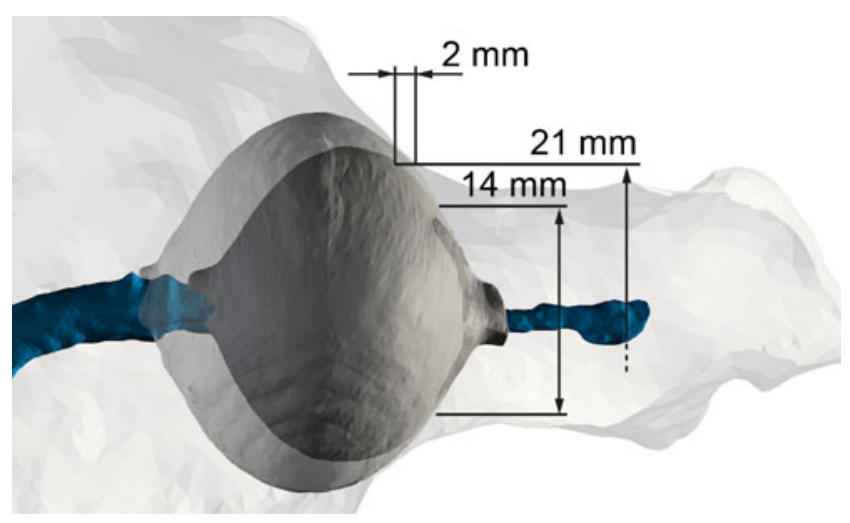

Fig. 4 Impact of balloon size and vein size on the site of ablation: overlay image of a scanned and 3D reconstructed $23 \mathrm{~mm}$ (inner dark gray) and $28 \mathrm{~mm}$ (outer semi-transparent) CB within a RSPV from a random patient undergoing $\mathrm{CB}$ ablation. Up to a diameter of approximately $14 \mathrm{~mm}$, the geometry of the two balloon sizes is almost identical. Consequently, ablation at the same ostial level can be expected. At a maximal usable diameter of the small $\mathrm{CB}$ of $21 \mathrm{~mm}$ (90\% of $\mathrm{CB}$ diameter), the freezing zone can be expected to be approximately $2 \mathrm{~mm}$ more distal in the PV compared to the $28 \mathrm{~mm}$ $\mathrm{CB}$. Due to the increasing curvature of the $23 \mathrm{~mm} \mathrm{CB}$ from this point on, a more distal ablation is likely. This may be problematic in large pulmonary veins sensitive warning sign of impending PNP and could prompt the operator to discontinue the freezing cycle prematurely. In all patients with PNP, the temperatures 30 and $40 \mathrm{~s}$ after initiation of the freezing cycle were below -41 and $-46{ }^{\circ} \mathrm{C}$, respectively. There was only one patient in the non-PNP group with temperatures below -41 and $-46{ }^{\circ} \mathrm{C}$ at the respective time points. This corresponds to a sensitivity of $100 \%$ and a specificity of $98 \%$ for low temperature as warning sign of PNP. It could be hypothesized that the mechanism behind this phenomenon is that in cases of very rapid temperature drop, there is good contact of the balloon over a larger surface area (allowing more energy transfer) and little contact of the balloon with the blood in the left atrium.

\subsection{Course of the phrenic nerve}

The anatomic proximity of the right $\mathrm{PN}$ and the right superior PV appears to be of paramount importance with regards to the development of PNP (Fig. 2). The distance between the anterior wall of the right superior PV and the $\mathrm{PN}$ in cadaver specimens is estimated to be approximately 2-3 mm. However, individual variation is considerable [10, $11,13]$. With a reported distance of $8-12 \mathrm{~mm}$ between the right inferior $\mathrm{PV}$ and the $\mathrm{PN}, \mathrm{CB}$ ablation at the right inferior PV probably has a very low risk of PNP. If PNP occurs with ablation at the right inferior PV, an anomaly of the course of the right $\mathrm{PN}$, of the right inferior $\mathrm{PV}$, or misinterpretation of the fluoroscopic information should be considered [10]. No PNP developed with ablation at the right inferior $\mathrm{PV}$ in our series, but this has been seen in other series [14].

Identification of the course of the PN by imaging the right pericardiophrenic artery using computed tomography has been described, but marked differences in its reliability have been reported between 20 and $100 \%$ [15, 16]. In contrast, the distance between the RSPV and the SVC as a marker of the proximity of the PN to the ablation site can easily be obtained from 3D reconstructions of the LA from computed tomography or magnetic resonance imaging often performed in clinical practice.

Although the RSPV-SVC distance was significantly different between the PNP and the non-PNP group, the force used to achieve adequate PV occlusion may result in anatomical distortion, and this may be an additional mechanism for PNP with CB ablation [17]. All procedures were performed by the same two main operators (MK and CS) using the same technique. However, forces applied during procedures could not be measured. The development of balloon catheters with novel integrated sensors on the curvilinear surfaces may overcome this limitation in the future and give detailed information on the $\mathrm{CB}$ surface temperature [18]. 


\subsection{Limitations}

This is a relatively small study. Whether the systematic use of this strategy has the potential to effectively prevent persistent PNP also in larger patient groups needs to be shown. A limitation of the protocol used is that diaphragmatic activity is manually and fluoroscopically assessed (not measured, e.g., by electromyography) and that it may be difficult to differentiate between loss of PN capture due to an unstable catheter position in the SVC and loss of capture due to acute, but reversible PNP [19]. It could be hypothesized that the three cases of PNP with immediate recovery of PN function in this study were due to dislodgement of the catheter in the SVC. This would mean that the rate of potential PN injuries is even overestimated in our study. However, loss of PN capture and subsequent recovery of PN function was observed without any obvious changes in catheter position and stable catheter positions were achieved, if necessary with the help of a deflectable catheter. Although performed in 3D, another limitation of this study is that the preprocedural measurements used to define the PVs (diameters at the ostium, ovality index, and perimeter) may be insufficient to adequately describe their complex anatomy during the procedure since potential distortions due to catheter manipulation are not taken into consideration. Finally, temperature measurements are obtained from a temperature probe at the proximal end of the $\mathrm{CB}$ and do not reflect the true temperature at the balloon-tissue interface.

\section{Conclusion}

The systematic use of PN stimulation in conjunction with a 28-mm CB ablation catheter cannot eliminate the problem of PNP during CB ablation of AF. However, the observed PNPs were only transient. The immediate termination of the freezing cycle in case of loss of capture and the more antral position of the $28 \mathrm{~mm} \mathrm{CB}$ catheter may play a key role here. The anatomical relationship between the right superior PV and the SVC was a predictor for the development of transient PNP, but left atrial and PV anatomy were not. This could have an impact on choosing an ablation strategy based on preprocedural imaging. Finally, low CB temperature early during the freezing cycle is a sensitive warning sign of impending PNP. Therefore, close monitoring of temperatures achieved during ablation at the right-sided PVs may further enhance the safety of the procedure.

\section{References}

1. Haissaguerre, M., Jais, P., Shah, D. C., Takahashi, A., Hocini, M., Quiniou, G., et al. (1998). Spontaneous initiation of atrial fibrillation by ectopic beats originating in the pulmonary veins. The New England Journal of Medicine, 339, 659-666.

2. Cappato, R., Calkins, H., Chen, S. A., Davies, W., Iesaka, Y., Kalman, J., et al. (2010). Updated worldwide survey on the methods, efficacy, and safety of catheter ablation for human atrial fibrillation. Circulation: Arrhythmia and Electrophysiology, 3, 32-38.

3. Calkins, H., Kuck, K. H., Cappato, R., Brugada, J., Camm, A. J., Chen, S. A., et al. (2012). 2012 HRS/EHRA/ECAS expert consensus statement on catheter and surgical ablation of atrial fibrillation. Heart Rhythm, 9, 632-696.

4. Van Belle, Y., Janse, P., Rivero-Ayerza, M. J., Thornton, A. S., Jessurun, E. R., Theuns, D., et al. (2007). Pulmonary vein isolation using an occluding cryoballoon for circumferential ablation: feasibility, complications, and short-term outcome. European Heart Journal, 28, 2231-2237.

5. Neumann, T., Vogt, J., Schumacher, B., Dorszewski, A., Kuniss, M., Neuser, H., et al. (2008). Circumferential pulmonary vein isolation with the cryoballoon technique results from a prospective 3-center study. Journal of the American College of Cardiology, 52, 273-278.

6. Chun, K. R., Schmidt, B., Metzner, A., Tilz, R., Zerm, T., Koster, I., et al. (2009). The 'single big cryoballoon' technique for acute pulmonary vein isolation in patients with paroxysmal atrial fibrillation: a prospective observational single centre study. European Heart Journal, 30, 699-709.

7. Kuhne, M., Suter, Y., Altmann, D., Ammann, P., Schaer, B., Osswald, S., et al. (2010). Cryoballoon versus radiofrequency catheter ablation of paroxysmal atrial fibrillation: biomarkers of myocardial injury, recurrence rates and pulmonary vein reconnection patterns. Heart Rhythm, 7, 1770-1776.

8. Packer, D., Irwin, J. M., Champagne, J., Guerra, P., Dubuc, M., Wheelan, K., et al. (2010). Cryoballoon ablation of pulmonary veins for paroxysmal atrial fibrillation: first results of the North American Arctic Front STOP-AF pivotal trial. Journal of the American College of Cardiology, 55, E3015E3016.

9. Andrade, J. G., Khairy, P., Guerra, P. G., Deyell, M. W., Rivard, L., Macle, L., et al. (2011). Efficacy and safety of cryoballoon ablation for atrial fibrillation: a systematic review of published studies. Heart Rhythm, 8, 1444-1451.

10. Sanchez-Quintana, D., Cabrera, J. A., Climent, V., Farre, J., Weiglein, A., \& Ho, S. Y. (2005). How close are the phrenic nerves to cardiac structures? Implications for cardiac interventionalists. Journal of Cardiovascular Electrophysiology, 16, 309-313.

11. Van Belle, Y., Knops, P., Janse, P., Rivero-Ayerza, M., Jessurun, E., Szili-Torok, T., et al. (2009). Electro-anatomical mapping of the left atrium before and after cryothermal balloon isolation of the pulmonary veins. Journal of Interventional Cardiac Electrophysiology, 25, 59-65.

12. Andrade, J., Dubuc, M., Guerra, P.G., Macle, L., Mondésert, B., Rivard, L., et al. (2012). The biophysics and biomechanics of cryoballoon ablation. Pacing and Clinical Electrophysiology, 35, $1162-1168$

13. Schmidt, B., Chun, K. R., Ouyang, F., Metzner, A., Antz, M., \& Kuck, K. H. (2008). Three-dimensional reconstruction of the anatomic course of the right phrenic nerve in humans by pace mapping. Heart Rhythm, 5, 1120-1126.

14. Kojodjojo, P., O’Neill, M. D., Lim, P. B., Malcolm-Lawes, L., Whinnett, Z. I., Salukhe, T. V., et al. (2010). Pulmonary venous isolation by antral ablation with a large cryoballoon for treatment of paroxysmal and persistent atrial fibrillation: mediumterm outcomes and non-randomised comparison with pulmonary venous isolation by radiofrequency ablation. Heart, 96, 13791384. 
15. Nieto-Tolosa, J., Rodriguez-Sanchez, D., Hurtado-Martinez, J.A., Pinar-Bermudez, E., Penafiel-Verdu, P., Sanchez-Munoz, J.J., et al. (2011). Phrenic nerve identification with cardiac multidetector computed tomography. Revista Española de Cardiología, 64, 942-944

16. Horton, R., Di Biase, L., Reddy, V., Neuzil, P., Mohanty, P., Sanchez, J., et al. (2010). Locating the right phrenic nerve by imaging the right pericardiophrenic artery with computerized tomographic angiography: implications for balloon-based procedures. Heart Rhythm, 7, 937-941.

17. Okumura, Y., Henz, B. D., Bunch, T. J., Dalegrave, C., Johnson, S. B., \& Packer, D. L. (2009). Distortion of right superior pulmonary vein anatomy by balloon catheters as a contributor to phrenic nerve injury. Journal of Cardiovascular Electrophysiology, 20, 1151-1157.

18. Kim, D. H., Lu, N., Ghaffari, R., Kim, Y. S., Lee, S. P., Xu, L., et al. (2011). Materials for multifunctional balloon catheters with capabilities in cardiac electrophysiological mapping and ablation therapy. Nature Materials, 10, 316-323.

19. Franceschi, F., Dubuc, M., Guerra, P. G., \& Khairy, P. (2011). Phrenic nerve monitoring with diaphragmatic electromyography during cryoballoon ablation for atrial fibrillation: the first human application. Heart Rhythm, 8, 1068-1071.

\section{Editorial Commentary}

Kuhne et al. evaluated whether a standardized protocol including phrenic nerve stimulation and the use of a large size $(28 \mathrm{~mm})$ balloon might prevent phrenic nerve palsy during Cryoballoon ablation of the right superior pulmonary vein. Although no case of persistent phrenic nerve palsy was reported, transient palsy occurred in $6 \%$ of patients. These findings support the notion that larger balloon sizes do not provide enough protection against the risk of phrenic nerve paralysis. Of note, the authors found that a short distance between the right superior pulmonary vein and the superior vena cava, and low temperature $(<41$ degrees Celsius) during the first 30 seconds of the freezing cycle were predictors of phrenic nerve palsy with very high sensitivity $(100 \%)$ and specificity $(98 \%)$. Further studies are warranted to define whether a pre-operative assessment of the distance between the right superior pulmonary vein and the superior vena cava might be useful to select patients with the lower risk of phrenic nerve palsy during Cryoballoon ablation of the right superior pulmonary vein. 